\title{
NUMERICAL SIMULATION OF RELATIVISTIC JET FORMATION IN BLACK HOLE MAGNETOSPHERE
}

\author{
SHINJI KOIDE \\ Faculty of Engineering, Toyama University, Gofuku, Toyama \\ 930, Japan \\ AND \\ KAZUNARI SHIBATA AND TAKAHIRO KUDOH \\ National Astronomical Observatory, Mitaka, Tokyo 181, Japan
}

\section{Introduction}

The radio jets ejected from active galactic nuclei (AGNs) sometimes show proper motions with apparent velocity exceeding the speed of light. This phenomenon, called superluminal motion, is explained as relativistic jets propagating in a direction almost toward us, and has been thought to be ejected from the close vicinity of hypothetical supermassive black holes powering AGNs (Rees 1996). The magnetic mechanism has been proposed not only for AGN jets (Lovelace 1976; Blandford \& Payne 1983) but also for protostellar jets (Pudritz \& Norman 1986; Uchida \& Shibata 1985; Shibata \& Uchida 1986), although no one has yet performed nonsteady general relativistic magnetohydrodynamic (GRMHD) numerical simulations on the formation of jets from the accretion disk around a black hole.

\section{Numerical Results}

We use $3+1$ formalism of general relativistic conservation laws of particle number, momentum, and energy and Maxwell equations with the infinite electric conductivity (Thorne et al. 1986; Koide et al. 1996; Koide 1997) to simulate the relativistic magnetohydrodynamic jet formation. The Schwarzschild metric, which provides the space-time around the black hole at rest, is used in the calculation. 
a

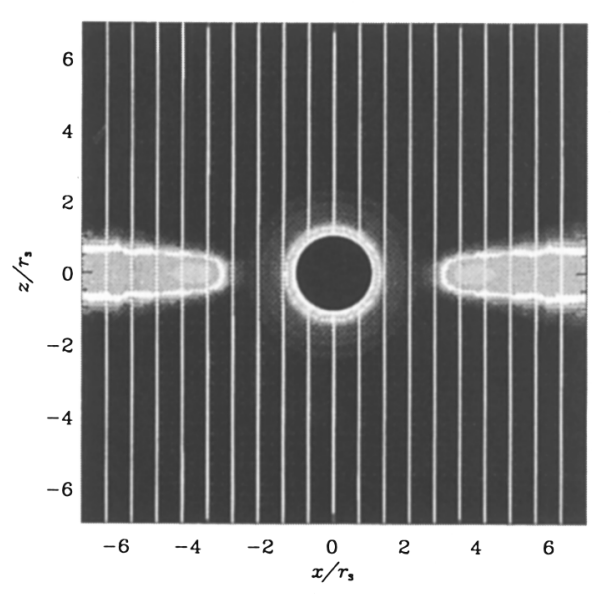

$\mathrm{b}$

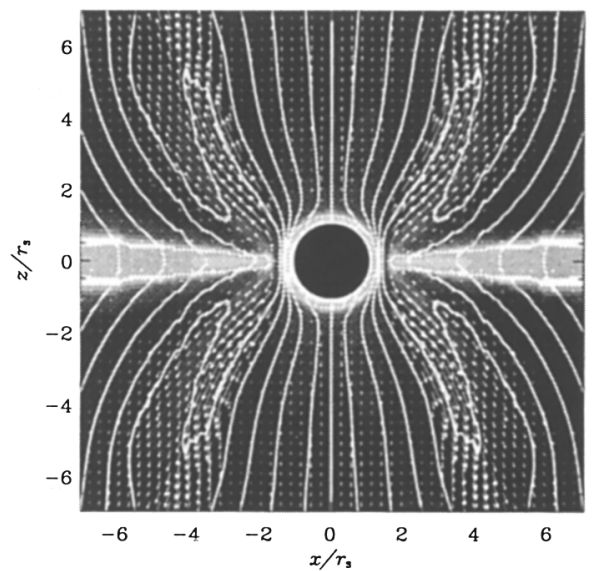

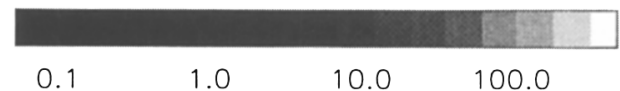

Figure 1. The jet formation in a black hole magnetosphere. (a) The initial condition. (b) $t=93 \tau_{\mathrm{S}}$.

Figure 1 shows the initial and final states of the relativistic jet formation in the black hole magnetosphere. The proper mass density (gray-scale), velocity (vector), and magnetic field (solid lines) are shown. The black circles at the centers represent the black hole inside the event horizon at Schwarzschild radius $r_{\mathrm{S}}$. Initially, the plasma of the corona is in hydrostatic equilibrium. The accretion disk rotates around the black hole with Keplerian velocity. The density of the disk is 400 times that of the background corona. The uniform magnetic field crosses the disk perpendicularly. Figure $1 \mathrm{~b}$ shows the final stage of this simulation at $t=91 \tau_{\mathrm{S}}$. The maximum poloidal component of the jet velocity is $0.88 \mathrm{c}$, which corresponds to a Lorentz factor of 2.1 at $x \sim 3 r_{\mathrm{S}}, z \sim 3 r_{\mathrm{S}}$. The jet has two-layered structure consisting of the inner, fast (low-density) jet and the outer, slow (high-density) jet. The former is ejected from the corona, while the latter is from the disk.

We thank M. Inda-Koide for her discussion and important comments for this study. We also thank M. Takahashi, T. Yokoyama, K. Hirotani, K.-I. Nishikawa, J.-I. Sakai, and R. L. Mutel for their discussion and encouragement. 